$£$ millions ${ }^{1}$

\begin{tabular}{|c|c|c|c|c|c|c|c|c|c|}
\hline & & & & & Net Borrowing & & & & Public sector \\
\hline & $\begin{array}{l}\text { surplus on } \\
\text { current budget }\end{array}$ & $\begin{array}{r}\text { Public sector } \\
\text { net investment }\end{array}$ & $\begin{array}{r}\text { Central } \\
\text { government }\end{array}$ & $\begin{array}{r}\text { Local } \\
\text { government }\end{array}$ & $\begin{array}{r}\text { General } \\
\text { government }\end{array}$ & $\begin{array}{r}\text { Public } \\
\text { corporations }\end{array}$ & Public sector & $\begin{array}{r}\text { Public sector } \\
\text { net debt }{ }^{2}\end{array}$ & $\begin{array}{r}\text { percentage of } \\
\text { GDP }\end{array}$ \\
\hline $\begin{array}{l}2001 \\
2002 \\
2003 \\
2004 \\
2005\end{array}$ & $\begin{array}{c}\text { ANMU } \\
19702 \\
-7000 \\
-18947 \\
-19648 \\
-17396\end{array}$ & $\begin{array}{r}\text {-ANNW } \\
11220 \\
12067 \\
15283 \\
17185 \\
24008\end{array}$ & 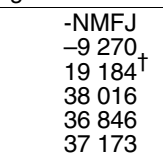 & $\begin{array}{r}-\mathrm{NMOE} \\
2724 \\
1205 \\
-785 \\
3188^{\dagger} \\
4226\end{array}$ & $\begin{array}{l}- \text { NNBK } \\
-6546 \\
20389 \\
37231 \\
40034 \\
41399\end{array}$ & $\begin{array}{r}- \text { IL6E } \\
-1936 \\
-1322 \\
-3001 \\
-3201 \\
5\end{array}$ & $\begin{array}{l}\text {-ANNX } \\
-8482 \\
19067 \dagger \\
34230 \\
36833 \\
41404\end{array}$ & $\begin{array}{l}\text { RUTN } \\
323.0 \\
348.1 \\
380.1 \\
424.0 \\
465.1\end{array}$ & $\begin{array}{r}\text { RUTO } \\
30.9 \\
31.5 \\
32.4 \\
34.6 \\
36.2\end{array}$ \\
\hline $\begin{array}{l}2001 / 02 \\
2002 / 03 \\
2003 / 04 \\
2004 / 05 \\
2005 / 06\end{array}$ & $\begin{array}{r}12117 \\
-11260 \\
-17433 \\
-18921 \\
-14025\end{array}$ & $\begin{array}{l}11901 \\
13804 \\
15701 \\
20724 \\
23746\end{array}$ & $\begin{array}{r}-183 \\
25403 \\
36335 \\
38135 \\
33276\end{array}$ & $\begin{array}{l}2081 \\
1074 \\
184 \\
3793 \\
5397\end{array}$ & $\begin{array}{r}1898 \\
26477 \\
36519 \\
41928 \\
38673\end{array}$ & $\begin{array}{r}-2114 \\
-1413 \\
-3385 \\
-2283 \\
-902\end{array}$ & $\begin{array}{r}-216 \\
25064 \\
33134 \\
39645 \\
37771\end{array}$ & $\begin{array}{l}314.3 \\
346.0 \\
381.5 \\
422.1 \\
461.6\end{array}$ & $\begin{array}{l}29.7 \\
30.8 \\
32.2 \\
34.1 \\
35.4\end{array}$ \\
\hline $\begin{array}{l}2006 / 07 \\
2007 / 08\end{array}$ & $\begin{array}{l}-4584 \\
-6066\end{array}$ & $\begin{array}{l}26027 \\
29505\end{array}$ & $\begin{array}{l}32152 \\
39158\end{array}$ & $\begin{array}{r}2795 \\
-249\end{array}$ & $\begin{array}{l}34947 \\
38909\end{array}$ & $\begin{array}{l}-4336 \\
-3338\end{array}$ & $\begin{array}{l}30611 \\
35571\end{array}$ & $\begin{array}{l}497.9 \\
621.2\end{array}$ & $\begin{array}{l}36.0 \\
43.0\end{array}$ \\
\hline $\begin{array}{r}2003 \text { Q1 } \\
\text { Q2 } \\
\text { Q3 } \\
\text { Q4 }\end{array}$ & $\begin{array}{r}6549 \\
-12148 \\
-4243 \\
-9105\end{array}$ & $\begin{array}{l}6362 \\
2188 \\
3349 \\
3384\end{array}$ & $\begin{array}{r}953 \\
16468 \\
7384 \\
13211\end{array}$ & $\begin{array}{r}-196 \\
-1441 \\
826 \\
26\end{array}$ & $\begin{array}{r}757 \\
15027 \\
8210 \\
13237\end{array}$ & $\begin{array}{l}-944 \\
-691 \\
-618 \\
-748\end{array}$ & $\begin{array}{r}-187 \\
14336 \\
7592 \\
12489\end{array}$ & $\begin{array}{l}346.0 \\
354.6 \\
359.8 \\
380.1\end{array}$ & $\begin{array}{l}30.8 \\
31.1 \\
31.1 \\
32.4\end{array}$ \\
\hline $\begin{array}{r}2004 \text { Q1 } \\
\text { Q2 } \\
\text { Q3 } \\
\text { Q4 }\end{array}$ & $\begin{array}{r}8063 \\
-12124 \\
-6126 \\
-9461\end{array}$ & $\begin{array}{l}6780 \\
2882 \\
3297 \\
4226\end{array}$ & $\begin{array}{r}-728 \\
16425 \\
8373 \\
12776\end{array}$ & $\begin{array}{l}{ }^{773} \\
-677^{\dagger} \\
1640 \\
1452\end{array}$ & $\begin{array}{rr} & 45 \\
15748 \\
10013 \\
14228\end{array}$ & $\begin{array}{r}-1328 \\
-742 \\
-590 \\
-541\end{array}$ & $\begin{array}{r}-1283 \\
15006 \\
9423 \\
13687\end{array}$ & $\begin{array}{l}381.5 \\
395.1 \\
401.4 \\
424.0\end{array}$ & $\begin{array}{l}32.2 \\
32.9 \\
33.1 \\
34.6\end{array}$ \\
\hline $\begin{array}{r}2005 \text { Q1 } \\
\text { Q2 } \\
\text { Q3 } \\
\text { Q4 }\end{array}$ & $\begin{array}{r}8790 \\
-11347 \\
-3507 \\
-11332\end{array}$ & $\begin{array}{r}10319 \\
2326 \\
4805 \\
6558\end{array}$ & $\begin{array}{r}561 \\
11757 \\
7231 \\
17624\end{array}$ & $\begin{array}{l}1378 \\
-996 \\
2318 \\
1526\end{array}$ & $\begin{array}{r}1939 \\
10761 \\
9549 \\
19150\end{array}$ & $\begin{array}{r}-410 \\
2912 \\
-1237 \\
-1260\end{array}$ & $\begin{array}{r}1529 \\
13673 \\
8312 \\
17890\end{array}$ & $\begin{array}{l}422.1 \\
437.4 \\
445.1 \\
465.1\end{array}$ & $\begin{array}{l}34.1 \\
34.9 \\
35.1 \\
36.2\end{array}$ \\
\hline $\begin{array}{r}2006 \text { Q1 } \\
\text { Q2 } \\
\text { Q3 } \\
\text { Q4 }\end{array}$ & $\begin{array}{r}12161 \\
-10770 \\
-1116 \\
-6831\end{array}$ & $\begin{array}{r}10057 \\
3961 \\
4852 \\
5068\end{array}$ & $\begin{array}{r}-3336 \\
21019 \\
5599 \\
11452\end{array}$ & $\begin{array}{r}2549 \\
-5425 \\
1214 \\
1805\end{array}$ & $\begin{array}{r}-787 \\
15594 \\
6813 \\
13257\end{array}$ & $\begin{array}{r}-1317 \\
-863 \\
-845 \\
-1358\end{array}$ & $\begin{array}{r}-2104 \\
14731 \\
5968 \\
11899\end{array}$ & $\begin{array}{l}461.6^{\dagger} \\
483.1 \\
486.2 \\
500.8\end{array}$ & $\begin{array}{l}35.4 \\
36.5 \\
36 .{ }^{\dagger} \\
36.8\end{array}$ \\
\hline $\begin{array}{r}2007 \text { Q1 } \\
\text { Q2 } \\
\text { Q3 } \\
\text { Q4 }\end{array}$ & $\begin{array}{r}14133 \\
-11912 \\
-933 \\
-9324\end{array}$ & $\begin{array}{r}12146^{\dagger} \\
2479 \\
5266 \\
6647\end{array}$ & $\begin{array}{r}-5918 \\
20540 \\
6341 \\
16052\end{array}$ & $\begin{array}{r}5201 \\
-5745 \\
607 \\
531\end{array}$ & $\begin{array}{r}-717 \\
14795 \\
6948 \\
16583\end{array}$ & $\begin{array}{r}-1270 \\
-404 \\
-749 \\
-612\end{array}$ & $\begin{array}{r}-1987 \\
14391 \\
6199 \\
15971\end{array}$ & $\begin{array}{l}497.9 \\
512.9 \\
514.5 \\
634.0\end{array}$ & $\begin{array}{l}36.0 \\
36.6 \\
36.2 \\
44.2\end{array}$ \\
\hline $\begin{array}{r}2008 \text { Q1 } \\
\text { Q2 } \\
\text { Q3 }\end{array}$ & $\begin{array}{r}16103 \\
-17929 \\
-7733\end{array}$ & $\begin{array}{r}15113 \\
4428 \\
6696\end{array}$ & $\begin{array}{l}-3775 \\
27763 \\
11808\end{array}$ & $\begin{array}{r}4358 \\
-5521 \\
2325\end{array}$ & $\begin{array}{r}583 \\
22242 \\
14133\end{array}$ & $\begin{array}{r}-1573 \\
115 \\
296\end{array}$ & $\begin{array}{r}-990 \\
22357 \\
14429\end{array}$ & $\begin{array}{l}621.2 \\
641.4 \\
644.5\end{array}$ & $\begin{array}{l}43.0 \\
44.0 \\
43.9\end{array}$ \\
\hline $\begin{array}{r}2006 \mathrm{Nov} \\
\text { Dec }\end{array}$ & $\begin{array}{l}-7167^{\dagger} \\
-3753\end{array}$ & $\begin{array}{l}1953 \\
1410\end{array}$ & $\begin{array}{l}9872^{\dagger} \\
4848\end{array}$ & $\begin{array}{l}367 \\
471\end{array}$ & $\begin{array}{c}10239^{\dagger} \\
5319\end{array}$ & $\begin{array}{c}-1119^{\dagger} \\
-156\end{array}$ & $\begin{array}{l}9120^{\dagger} \\
5163\end{array}$ & $\begin{array}{l}486.6^{\dagger} \\
500.8\end{array}$ & $\begin{array}{l}35.9^{\dagger} \\
36.8\end{array}$ \\
\hline $\begin{array}{r}2007 \text { Jan } \\
\text { Feb } \\
\text { Mar } \\
\text { Apr } \\
\text { May } \\
\text { Jun }\end{array}$ & $\begin{array}{r}13474 \\
3229 \\
-2570 \\
397 \\
-7097 \\
-5212\end{array}$ & $\begin{array}{c}3816^{\dagger} \\
4010 \\
4320 \\
47 \\
1515 \\
917\end{array}$ & $\begin{array}{r}-11169 \\
-317 \\
5568 \\
2765 \\
11306 \\
6469\end{array}$ & $\begin{array}{r}1887^{\dagger} \\
1823 \\
1491 \\
-3416 \\
-2035 \\
-294\end{array}$ & $\begin{array}{r}-9282 \\
1506 \\
7059 \\
-651 \\
9271 \\
6175\end{array}$ & $\begin{array}{r}-376 \\
-725 \\
-169 \\
301 \\
-659 \\
-46\end{array}$ & $\begin{array}{r}-9658 \\
781 \\
6890 \\
-350 \\
8612 \\
6129\end{array}$ & $\begin{array}{l}478.9 \\
480.5 \\
497.9 \\
494.4 \\
501.7 \\
512.9\end{array}$ & $\begin{array}{l}35.0 \\
34.9 \\
36.0 \\
35.6 \\
36.0 \\
36.6\end{array}$ \\
\hline $\begin{array}{l}\text { Jul } \\
\text { Aug } \\
\text { Sep } \\
\text { Oct } \\
\text { Nov } \\
\text { Dec }\end{array}$ & $\begin{array}{r}8147 \\
-6205 \\
-2875 \\
2922 \\
-8124 \\
-4122\end{array}$ & $\begin{array}{r}1780 \\
1608 \\
1878 \\
866 \\
2532 \\
3249\end{array}$ & $\begin{array}{r}-6514 \\
6882 \\
5973 \\
-1928 \\
10995 \\
6985\end{array}$ & $\begin{array}{r}761 \\
1185 \\
-1339 \\
373 \\
144 \\
14\end{array}$ & $\begin{array}{r}-5753 \\
8067 \\
4634 \\
-1555 \\
11139 \\
6999\end{array}$ & $\begin{array}{r}-614 \\
-254 \\
119 \\
-501 \\
-483 \\
372\end{array}$ & $\begin{array}{r}-6367 \\
7813 \\
4753 \\
-2056 \\
10656 \\
7371\end{array}$ & $\begin{array}{l}499.5 \\
506.8 \\
514.5 \\
607.1 \\
617.1 \\
634.0\end{array}$ & \begin{tabular}{l|l}
35.5 \\
35.8 \\
36.2 \\
42.6 \\
43.1 \\
44.2
\end{tabular} \\
\hline $\begin{array}{r}2008 \text { Jan } \\
\text { Feb } \\
\text { Mar } \\
\text { Apr } \\
\text { May } \\
\text { Jun }\end{array}$ & $\begin{array}{r}15087 \\
4423 \\
-3407 \\
-1176 \\
-10922 \\
-5831\end{array}$ & $\begin{array}{r}1428 \\
5478 \\
8207 \\
665 \\
2065 \\
1698\end{array}$ & $\begin{array}{r}-15779 \\
1267 \\
10737 \\
5423 \\
13522 \\
8818\end{array}$ & $\begin{array}{r}2331 \\
1654 \\
373 \\
-2862 \\
-988 \\
-1671\end{array}$ & $\begin{array}{r}-13448 \\
2921 \\
11110 \\
2561 \\
12534 \\
7147\end{array}$ & $\begin{array}{r}-211 \\
-1866 \\
504 \\
-720 \\
453 \\
382\end{array}$ & $\begin{array}{r}-13659 \\
1055 \\
11614 \\
1841 \\
12987 \\
7529\end{array}$ & $\begin{array}{l}607.8 \\
610.4 \\
621.2 \\
618.8 \\
629.1 \\
641.4\end{array}$ & $\begin{array}{l}42.2 \\
42.3 \\
43.0 \\
42.7 \\
43.3 \\
44.0\end{array}$ \\
\hline $\begin{array}{l}\text { Jul } \\
\text { Aug } \\
\text { Sep } \\
\text { Oct } \\
\text { Nov }\end{array}$ & $\begin{array}{r}6711 \\
-8422 \\
-6022 \\
-687 \\
-13046\end{array}$ & $\begin{array}{l}2381 \\
1874 \\
2441 \\
2597 \\
2951\end{array}$ & $\begin{array}{r}-5142 \\
9336 \\
7614 \\
3325 \\
15632\end{array}$ & $\begin{array}{l}881 \\
956 \\
488 \\
930 \\
977\end{array}$ & $\begin{array}{r}-4261 \\
10292 \\
8102 \\
4255 \\
16609\end{array}$ & $\begin{array}{r}-69 \\
4 \\
361 \\
-971 \\
-612\end{array}$ & $\begin{array}{r}-4330 \\
10296 \\
8463 \\
3284 \\
15997\end{array}$ & $\begin{array}{l}627.2 \\
632.7 \\
644.5 \\
640.6 \\
650.0\end{array}$ & $\begin{array}{l}42.9 \\
43.2 \\
43.9 \\
43.6 \\
44.2\end{array}$ \\
\hline
\end{tabular}




\begin{tabular}{|c|c|c|c|c|c|c|c|c|c|c|}
\hline & \multicolumn{10}{|c|}{ Current receipts } \\
\hline & \multirow{2}{*}{$\begin{array}{c}\text { Taxes on } \\
\text { production } \\
\text { Total }\end{array}$} & \multirow{2}{*}{$\begin{array}{r}\text { of which } \\
\text { VAT }\end{array}$} & \multicolumn{3}{|c|}{ Taxes on income and wealth } & \multirow{2}{*}{\multicolumn{2}{|c|}{$\begin{array}{r}\text { Compulsory } \\
\text { social } \\
\text { contributions }\end{array}$}} & \multirow[b]{2}{*}{$\begin{array}{r}\text { Interest and } \\
\text { dividends }\end{array}$} & \multirow[b]{2}{*}{$\begin{array}{r}\text { Other } \\
\text { receipts }^{3}\end{array}$} & \multirow[b]{2}{*}{ Total } \\
\hline & & & Total & $\begin{array}{r}\text { Income and } \\
\text { capital gains } \\
\operatorname{tax}^{1}\end{array}$ & Other ${ }^{2}$ & & & & & \\
\hline & NMBY & NZGF & NMCU & LIBR & LIBP & LIQR & AllH & LIQP & LIQQ & ANBV \\
\hline $2002 / 03$ & 140152 & 69087 & 143256 & 112373 & 30883 & 9588 & 63529 & 7918 & 7232 & 371675 \\
\hline $2003 / 04$ & 148758 & 76638 & 145487 & 115233 & 30254 & 10309 & 75148 & 7794 & 7166 & 394662 \\
\hline $2004 / 05$ & 154963 & 79978 & 160490 & 124477 & 36013 & 10950 & 80923 & 7466 & 7302 & 422094 \\
\hline $2005 / 06$ & 159254 & 81496 & 179960 & 134918 & 45042 & 11760 & 85559 & 7749 & 7529 & 451811 \\
\hline $2006 / 07$ & 169897 & 87735 & 194499 & 146779 & 47720 & 12520 & 90818 & 7975 & 7543 & 483252 \\
\hline $2007 / 08$ & 175807 & 89878 & 207840 & 158499 & 49341 & 13267 & 95510 & 9978 & 7755 & 510157 \\
\hline 2007 Feb & $13484^{\dagger}$ & $6790^{\dagger}$ & 19242 & 17720 & 1522 & 1034 & 7992 & $611^{\dagger}$ & 633 & 42996 \\
\hline Mar & 14719 & 7804 & 12874 & 11980 & 894 & 1211 & 9806 & 978 & $630^{\dagger}$ & 40218 \\
\hline Apr & 14792 & 7707 & $16316^{\dagger}$ & $10286^{\dagger}$ & 6030 & 1089 & $7138^{\dagger}$ & 830 & 672 & $40837^{\dagger}$ \\
\hline Jun & 14733 & 7303 & 11817 & 10387 & 1430 & 1114 & 7758 & 589 & 670 & 36681 \\
\hline Jul & 14909 & 7575 & 25578 & 15616 & 9962 & 1125 & 7508 & 702 & 634 & 50456 \\
\hline Aug & 14777 & 7267 & 12608 & 11766 & 842 & 1196 & 7310 & 671 & 633 & 37195 \\
\hline Sep & 15023 & 8006 & 12927 & 10523 & 2404 & 1118 & 7695 & 778 & 634 & 38175 \\
\hline Oct & 15141 & 7586 & 20354 & 10606 & 9748 & 1137 & 7472 & 1007 & 645 & 45756 \\
\hline Nov & 14919 & 7520 & 11884 & 10326 & 1558 & 1108 & 7583 & 911 & 645 & 37050 \\
\hline Dec & 14695 & 7622 & 14303 & 11862 & 2441 & 960 & 8203 & 895 & 648 & 39704 \\
\hline 2008 Jan & 13829 & 7202 & 35795 & 25473 & 10322 & 1052 & 8315 & 839 & 642 & 60472 \\
\hline Feb & 14086 & 7054 & 19228 & 17866 & 1362 & 1166 & 9208 & 791 & 642 & 45121 \\
\hline Mar & 14416 & 7677 & 15707 & 13593 & 2114 & 1094 & 10149 & 1251 & 617 & 43234 \\
\hline Apr & 15420 & 8283 & 16819 & 10313 & 6506 & 1196 & 7891 & 807 & 655 & 42788 \\
\hline May & 14877 & 7857 & 10641 & 9576 & 1065 & 1038 & 7723 & 695 & 656 & 35630 \\
\hline Jun & 15019 & 8061 & 11917 & 10445 & 1472 & 1038 & 8317 & 690 & 655 & 37636 \\
\hline Jul & 14709 & 7684 & 26198 & 16081 & 10117 & 1175 & 7707 & 720 & 657 & 51166 \\
\hline Aug & 14000 & 7196 & 13072 & 11973 & $1099^{\dagger}$ & 1020 & 7733 & 711 & 660 & 37196 \\
\hline Sep & 14598 & 7844 & 13531 & 10435 & 3096 & 1062 & 7981 & 992 & 657 & 38821 \\
\hline Oct & 14704 & 7694 & 19888 & 9998 & 9890 & 1143 & 7715 & 857 & 674 & 44981 \\
\hline \multirow{2}{*}{ Nov } & & Net Social & & & lus capital & & Surplus on & \multirow[b]{2}{*}{ Net investment } & \multirow{2}{*}{\multicolumn{2}{|c|}{ Net borrowing }} \\
\hline & Interest & Benefits & Other & Total & taxes & Depreciation & current budget & & & \\
\hline & NMFX & GZSJ & LIQS & ANLP & ANPM & NSRN & ANLV & -ANNS & & $\overline{-N M F J}$ \\
\hline $2002 / 03$ & 20911 & 109144 & 244547 & 374602 & -2927 & 5194 & -8121 & 17282 & & 25403 \\
\hline $2003 / 04$ & 22280 & 116926 & 267583 & 406789 & -12127 & 5514 & -17641 & 18694 & & 36335 \\
\hline $2004 / 05$ & 23933 & 122624 & 288019 & 434576 & -12482 & 5513 & -17995 & 20140 & & 38135 \\
\hline $2005 / 06$ & 25807 & 127304 & 306813 & 459924 & -8113 & 5734 & -13847 & 19429 & & 33276 \\
\hline $2006 / 07$ & 27576 & 131347 & 324185 & 483108 & 144 & 5881 & -5737 & 26415 & & 32152 \\
\hline $2007 / 08$ & 29943 & 140174 & 340703 & 510820 & -663 & 6133 & -6796 & 32362 & & 39158 \\
\hline 2007 Feb & 2715 & 9907 & $26382^{\dagger}$ & $39004^{\dagger}$ & $3992^{\dagger}$ & 488 & $3504^{\dagger}$ & $3187^{\dagger}$ & & $-317^{\dagger}$ \\
\hline Mar & 1597 & 11212 & 29007 & 41816 & -1598 & 488 & -2086 & 3482 & & 5568 \\
\hline Apr & 2560 & 10930 & 28974 & 42464 & -1627 & 507 & -2134 & 631 & & 2765 \\
\hline May & 2818 & 11700 & 29448 & 43966 & -8490 & 507 & -8997 & 2309 & & 11306 \\
\hline Jun & 2013 & 11567 & 27290 & 40870 & -4189 & 506 & -4695 & 1774 & & 6469 \\
\hline Jul & 2876 & 11636 & 26988 & 41500 & 8956 & 510 & 8446 & 1932 & & -6514 \\
\hline Aug & 3080 & 11802 & 26864 & 41746 & -4551 & 510 & -5061 & 1821 & & 6882 \\
\hline Sep & 1043 & 11629 & 28851 & 41523 & -3348 & 509 & -3857 & 2116 & & 5973 \\
\hline Oct & 3043 & 11791 & 27042 & 41876 & 3880 & 523 & 3357 & 1429 & & -1928 \\
\hline Nov & 3023 & 13589 & 28148 & 44760 & -7710 & 523 & -8233 & 2762 & & 10995 \\
\hline Dec & 2424 & 11641 & 28656 & 42721 & -3017 & 524 & -3541 & 3444 & & 6985 \\
\hline 2008 Jan & 3065 & 11767 & 28248 & 43080 & 17392 & 505 & 16887 & 1108 & & -15779 \\
\hline Feb & 3038 & 11197 & 26507 & 40742 & 4379 & 504 & 3875 & 5142 & & 1267 \\
\hline Mar & 960 & 10925 & 33687 & 45572 & -2338 & 505 & -2843 & 7894 & & 10737 \\
\hline Apr & $3156^{\dagger}$ & $12221^{\dagger}$ & 30886 & 46263 & -3475 & $525^{\dagger}$ & -4000 & 1423 & & 5423 \\
\hline May & 2943 & 12536 & 30526 & 46005 & -10375 & 525 & -10900 & 2622 & & 13522 \\
\hline Jun & 2489 & 12164 & 28986 & 43639 & -6003 & 526 & -6529 & 2289 & & 8818 \\
\hline Jul & 3293 & 12681 & 27445 & 43419 & 7747 & 526 & 7221 & 2079 & & -5142 \\
\hline Aug & 3385 & 12559 & 28341 & 44285 & -7089 & 526 & -7615 & 1721 & & 9336 \\
\hline Sep & 1301 & 12521 & 29816 & 43638 & -4817 & 527 & -5344 & 2270 & & 7614 \\
\hline Oct & 3394 & 12039 & 29337 & 44770 & 211 & 527 & -316 & 3009 & & 3325 \\
\hline Nov & 3146 & 14556 & 29849 & 47551 & -12443 & 527 & -12970 & 2662 & & 15632 \\
\hline
\end{tabular}

1 Includes capital gains tax paid by households. Includes income tax and cap- 3 Includes receipts from the spectrum.

ital gains tax paid by corporations.

2 Mainly comprises corporation tax and petroleum revenue tax.

Source: Office for National Statistics: 02070142129 
Government finance

\subsection{Public sector aggregates ${ }^{1}$}

$£$ millions, not seasonally adjusted

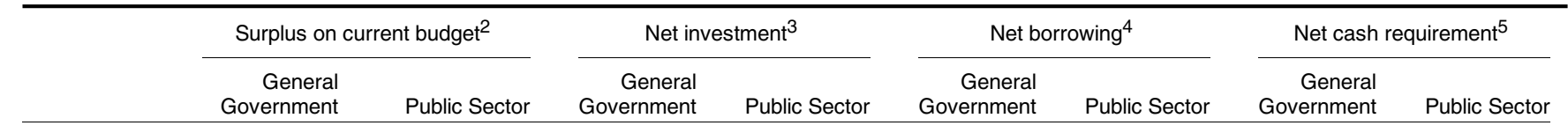

\section{Calendar years}

2001

2002

2004

2005

2006

2007

ANLW
19879
-6145
-17958
-18239
-18995
$-11545^{\dagger}$
-13982

ANMU
19702
$-7000^{\dagger}$
-18947
-19648
-17396
-6556
-8036

-ANNV
10088
11132
18435
19575
20599
$23321^{\dagger}$
23626

$\begin{array}{rl}\text {-ANNW } & \text {-NNBK } \\ 11220 & -6546 \\ 12067 & 20389^{\dagger} \\ 15283 & 37231 \\ 17185 & 40034 \\ 24008 & 41399 \\ & \\ 23938 & 34877 \\ 26538^{\dagger} & 37609\end{array}$

Financial years

$2001 / 02$
$2002 / 03$
$2003 / 04$
$2004 / 05$
$2005 / 06$
$2006 / 07$
$2007 / 08$

$$
\begin{array}{r}
10640 \\
-9734 \\
-16611 \\
-17878 \\
-17482 \\
-9742 \\
-11586
\end{array}
$$
12117
$-11260$
$-17433$
$-18921$
$-14025$
$-4584$
$-6066$

11555
13406
18005
21238
18693
24794
27602

11901
13804
15701
20724
23746
26027
29505

1898
26477
36519
41928
38673
34947
38909

-216
25064
33134
39645
37771
30611
35571

-ANNX
-8482
$19067^{\dagger}$
34230
36833
41404
30494
34574

\section{RUUS}

$-3768$

16421

38214

41311

$-1985$

19121

37234

36648

$30787^{\dagger}$

33412

$32257^{\dagger}$

\section{Quarterly}

2000 Q4

$$
2664^{\dagger}
$$

2556
18394
-2994
5063

$1784^{\dagger}$

1066
3944
1641

3283

18876
-2748

4871

Q3
Q4

$-1120$

$-761$

2251

3034

1641

2355
3280

$\begin{array}{ll}10809 & 4750 \\ -9887^{\dagger} & 1297\end{array}$

$-8898$

-313
-6571

-815
-7107

1297
2983

2983
2102

4625
1148

2747

3547

6549

6048

2003 Q1

$$
\text { Q2 }
$$

Q4

$-11115$

-3392
-9499

$-12148$

$-4243$

7024
3135

4033

6362

2188

3349

3384

8063

7395

$-10936$

-5068
-9630

$-12124$

$-6126$

$-9461$

\section{4}

3800

4279

6780

2882

3297

4226

$8790 \quad 8257$

7756

$-10488$

-3888
-12375

$-11347$

-11347
-3507

8257
2269

$$
\begin{aligned}
& 4716 \\
& 5357
\end{aligned}
$$

10319

2326

4805
6558

-739
-13775
5110

5110
-2250

4369

-5331
$11473^{\dagger}$

4134

10113

757
15027

8210

13237

$-11332$

$$
6351
$$

9269

$-11385$

-2130
-8080

12161

-10770
-1116

$-6831$

3617

4667
5224

10057

3961

4852

5068

45
15748

15748
10013

14228

$\begin{array}{rr}14133 & 11286 \\ -11912 & 2107 \\ -933 & 4348\end{array}$

$-9324$

$12146^{\dagger}$

2479
5266

6647

1939

10761

9549

19150

5885

$-787$

15594

6813

13257

-1490
-14450
4635
-2708
4041
-6184
$11035^{\dagger}$
3562

10654

-187
14336

7592

12489

2943
21499
40005
38724
40061

36949
28898

4078

24562

38448

38421

40396

35110

21572

-717
14795

$\begin{array}{rr}6948 & 6199 \\ 16583 & 15971\end{array}$

$-1283$

15006

9423

13687

4187

3606

$-13094$

6246
-6322

9402

$-11724$

6347

$-6107$

9499

$-6383$

7126

$-145$

$-5661$

-5661
6698

1243

16841

$-1305$

16404

6036

$-220$

-220
15275

5814

16365

$\begin{array}{rr}486 & 994 \\ 11577 & 11987\end{array}$

$\begin{array}{rr}6968 & 7034 \\ 22280 & 22182\end{array}$

$\begin{array}{rrr}1529 & -2101 & -2782 \\ 13673 & 15947 & 16297\end{array}$

13673

8312

15947
8464

17890

19556

8239

19318

$-2104$

14731
5968

$\begin{array}{ll}-3906 & -3458 \\ 19274 & 18969\end{array}$

$\begin{array}{rr}19274 & 18969 \\ 5600 & 5520\end{array}$

$\begin{array}{rr}15680 & 12381\end{array}$

11899

$-3605$

-1987
14391

12957

20859

$-1760$

15971

20859

$-990$

$15113-583$

15262
3677

3677
5636

$\begin{array}{ll}4428 & 22242 \\ 6696 & 14133\end{array}$

14133

22357
14429

$-5494$

25953
22422

12641
$727^{\dagger}$

20649

$-7733$

1 National accounts entitie

4 Net borrowing $=$ net investment minus surplus on current budget

counts 1995 (ESA95)

5 Previously called Public Sector Borrowing Requirement (PSBR).

2 Net saving, plus capital taxes.

3 Gross capital formation, plus payments less receipts, of investment grants

Source: Office for National Statistics: 02070142129

less depreciation. 


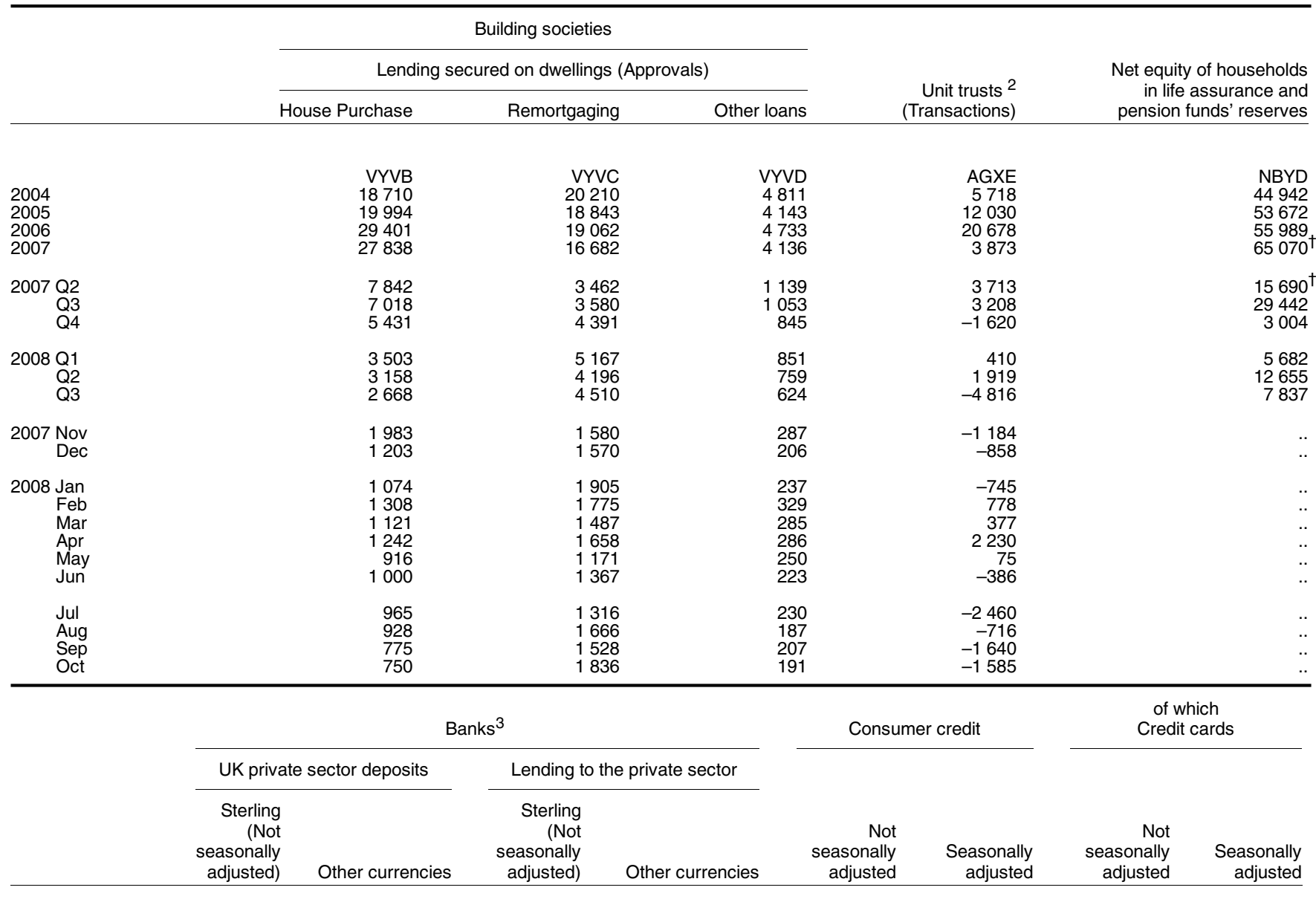

Amount outstanding

as at $31 \mathrm{Dec}$

2007

\begin{tabular}{rrrr} 
AEAS & AGAK & AECE & AECK \\
1440209 & $394771^{\dagger}$ & 1836074 & 522653 \\
\hline
\end{tabular}

Transactions

$\begin{array}{rrrr} & \text { AEAT } & \text { AEAZ } & \text { AECF } \\ 2004 & 86098 & 27697 & 133774 \\ 2005 & 137241 & 39735 & 137555 \\ 2006 & 150052 & 58434 & 191019 \\ 2007 & 156159^{\dagger} & 71686^{\dagger} & 213352 \\ 2007 \text { Q2 } & 50845^{\dagger} & 40067^{\dagger} & 51343 \\ \text { Q3 } & 38402 & -2836 & 81473 \\ \text { Q4 } & 25847 & 12323 & 22767 \\ & & & \\ 2008 \text { Q1 } & 32276 & 38988 & 50414^{\dagger} \\ \text { Q2 } & 55655 & -25028 & 93771 \\ \text { Q3 } & 47591 & -27955 & 59983 \\ & & & \\ 2007 \text { Dec } & 23637^{\dagger} & -19358^{\dagger} & 5224 \\ \text { 2008 Jan } & 5527 & 25093 & 27556^{\dagger} \\ \text { Feb } & 2445 & 8022 & 12643 \\ \text { Mar } & 24304 & 5873 & 10215 \\ \text { Apr } & 3371 & 9127 & 31223 \\ \text { May } & 10373 & -4131 & 4096 \\ \text { Jun } & 41911 & -30024 & 58452 \\ & & & \\ \text { Jul } & -3067 & -1921 & 9145 \\ \text { Aug } & 27613 & -4416 & 25264 \\ \text { Sep } & 23045 & -21618 & 25574 \\ \text { Oct } & 53120 & 8375 & 46897 \\ \text { Nov } & & & \end{array}$

$$
\text { Nov }
$$

8375

1 For further details see Financial Statistics, Tables 1.2E, 3.2B, 4.2A, 4.3A,

4.3B, 5.2D, 6.2A, 10.5D.

2 Including open ended investment companies (OEICs).

3 Monthly figures relate to calendar months.

\begin{tabular}{|c|c|c|c|}
\hline Net lending & Net lending & Net lending & Net lending \\
\hline $\begin{array}{c}\text { VZQC } \\
25337 \\
19666 \\
13054 \\
13490\end{array}$ & $\begin{array}{c}\mathrm{RLMH}^{\mathrm{RL}} \mathrm{H}^{\dagger} \\
19801 \\
13276 \\
13206\end{array}$ & $\begin{array}{l}\text { VZQS } \\
9998 \\
6166 \\
1951 \\
2251\end{array}$ & $\begin{array}{l}\text { VZQX } \\
9898 \\
6109 \\
2063 \\
2060\end{array}$ \\
\hline $\begin{array}{l}3951 \\
3948 \\
4049\end{array}$ & $\begin{array}{l}3272^{\dagger} \\
3982 \\
3602\end{array}$ & $\begin{array}{r}598 \\
941 \\
2033\end{array}$ & $\begin{array}{l}244^{\dagger} \\
835 \\
786\end{array}$ \\
\hline $\begin{array}{l}3 \text { 3 } 325^{\dagger} \\
38882 \\
2375\end{array}$ & $\begin{array}{l}4147 \\
3190 \\
2201\end{array}$ & $\begin{array}{l}-714 \\
1524 \\
1464\end{array}$ & $\begin{array}{r}966 \\
1185 \\
1154\end{array}$ \\
\hline 982 & $669^{\dagger}$ & 1375 & $286^{\dagger}$ \\
\hline $\begin{array}{c}777 \\
1439 \\
1110 \\
1439 \\
1546 \\
897\end{array}$ & $\begin{array}{r}829 \\
1817 \\
1501 \\
953 \\
1182 \\
1055\end{array}$ & $\begin{array}{r}-737 \\
18 \\
5 \\
297 \\
656 \\
571\end{array}$ & $\begin{array}{l}226 \\
344 \\
397 \\
152 \\
603 \\
429\end{array}$ \\
\hline $\begin{array}{r}663 \\
470 \\
1242 \\
354 \\
418\end{array}$ & $\begin{array}{r}853 \\
1017 \\
330 \\
754 \\
751\end{array}$ & $\begin{array}{l}173 \\
717 \\
573 \\
-44 \\
659\end{array}$ & $\begin{array}{l}248 \\
627 \\
279 \\
405 \\
396\end{array}$ \\
\hline
\end{tabular}

Sources: Office for National Statistics; Department for National Savings; Association of Unit Trusts and Investment Funds; Bank of England;
Department for Business, Enterprise and Regulatory Reform 


\begin{tabular}{|c|c|c|c|c|c|c|}
\hline & \multicolumn{6}{|c|}{ Amount outstanding } \\
\hline & \multicolumn{2}{|c|}{$\begin{array}{c}\text { Notes and coin in circulation outside the } \\
\text { Bank of England }\end{array}$} & \multicolumn{2}{|c|}{ Retail deposits and cash in M4 } & \multicolumn{2}{|r|}{ M4 } \\
\hline & $\begin{array}{r}\text { Not } \\
\text { seasonally } \\
\text { adjusted } \\
\end{array}$ & Seasonally adjusted & $\begin{array}{r}\text { Not } \\
\text { seasonally } \\
\text { adjusted }\end{array}$ & Seasonally adjusted & $\begin{array}{r}\text { Not } \\
\text { seasonally } \\
\text { adjusted } \\
\end{array}$ & Seasonally adjusted \\
\hline & AVAA & AVAB & VQXV & VQWU & AUYM & AUYN \\
\hline 2004 & 41147 & 41176 & 845654 & $843162^{\dagger}$ & 1179192 & $1173215^{\dagger}$ \\
\hline 2005 & 43034 & 43001 & 922687 & 920652 & 1328321 & 1321846 \\
\hline 2006 & 45213 & 45198 & 996629 & 994047 & 1498920 & 1490455 \\
\hline 2007 & 47361 & 47325 & 1072003 & 1070456 & 1676182 & 1669021 \\
\hline 2006 Q2 & 44876 & $45027^{\dagger}$ & 962858 & $955558^{\dagger}$ & 1420482 & $1408835^{\dagger}$ \\
\hline Q3 & 45473 & 45533 & 973901 & 972697 & 1460436 & 1456815 \\
\hline Q4 & 46560 & 45838 & 996629 & 994047 & 1498920 & 1490455 \\
\hline 2007 Q1 & 45738 & 46229 & 1011748 & 1007523 & 1543167 & 1536995 \\
\hline Q4 & 49212 & 48359 & 1072003 & 1070456 & 1676182 & 1669021 \\
\hline 2008 Q1 & 49102 & 49251 & 1109502 & 1104175 & $1721133^{\dagger}$ & 1716761 \\
\hline Q2 & 49490 & 49852 & 1127625 & 1117219 & 1780053 & 1771319 \\
\hline Q3 & 50178 & 50268 & $1127391^{\dagger}$ & 1126558 & 1845835 & 1842865 \\
\hline 2007 Oct & 47985 & $48198^{\dagger}$ & 1051344 & $1057304^{\dagger}$ & 1644623 & $1644534^{\dagger}$ \\
\hline Nov & 48634 & 48350 & 1060650 & 1060761 & 1650418 & 1643905 \\
\hline Dec & 51016 & 48528 & 1072003 & 1070456 & 1676182 & 1669021 \\
\hline 2008 Jan & 49461 & 48919 & 1078399 & 1089922 & $1683084^{\dagger}$ & 1695910 \\
\hline Feb & 48355 & 49259 & 1082576 & 1093685 & 1691423 & 1701931 \\
\hline Mar & 49491 & 49576 & 1109502 & 1104175 & 1721133 & 1716761 \\
\hline Apr & 49124 & 49697 & 1113224 & 1109917 & 1726998 & 1728187 \\
\hline May & 49697 & 49835 & 1122408 & 1115357 & 1738822 & 1736995 \\
\hline Jun & 49649 & 50023 & 1127625 & 1117219 & 1780053 & 1771319 \\
\hline Jul & 50030 & 50160 & 1117957 & 1118093 & 1776656 & 1782828 \\
\hline Aug & 50229 & 50103 & 1123020 & 1121657 & 1805641 & 1810919 \\
\hline
\end{tabular}

Source: Bank of England

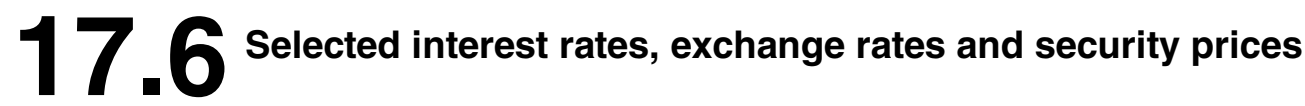

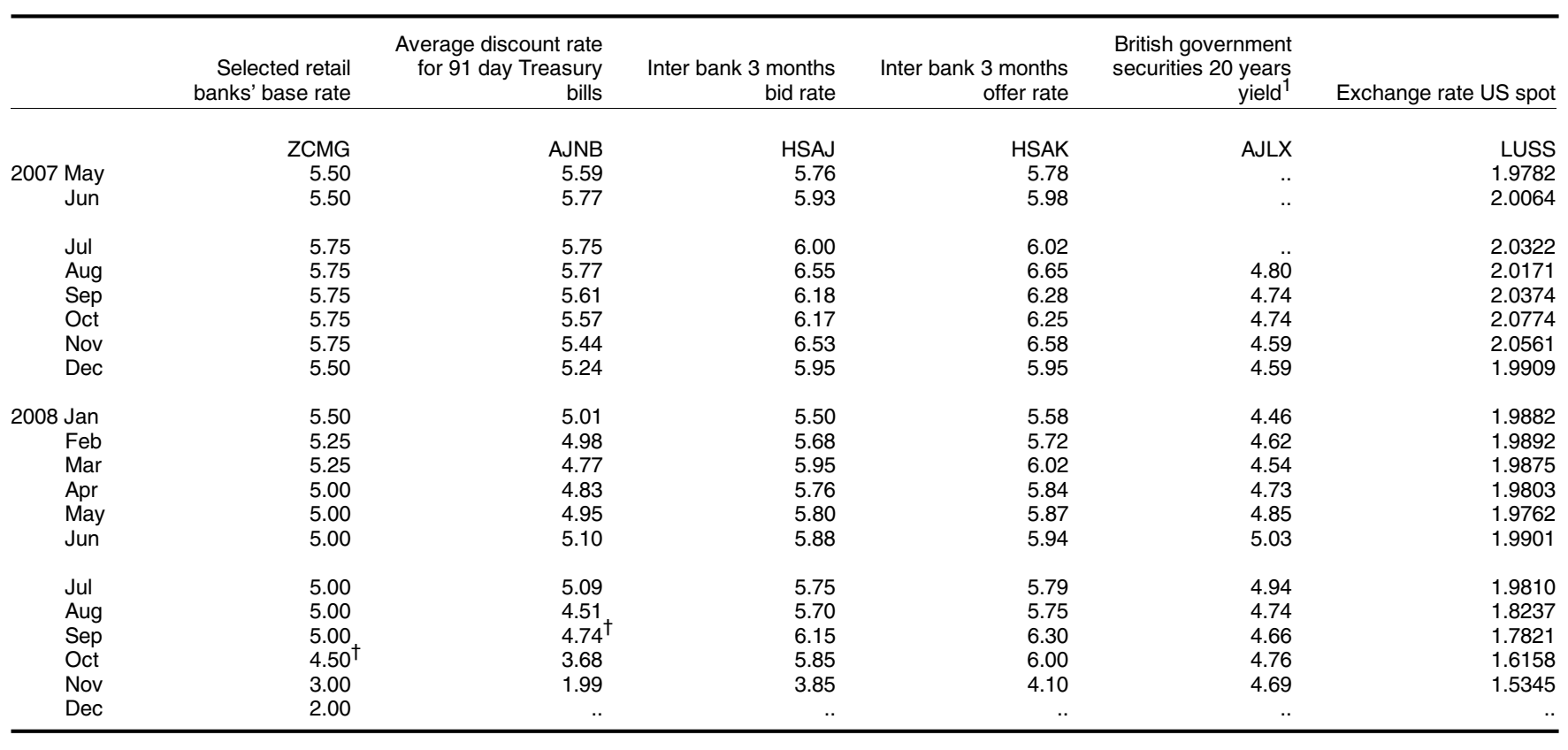

